\title{
Efectividad de la Remoción Parcial de Caries en Molares Primarios con Lesiones de Caries Profunda. Ensayo Clínico Aleatorizado
}

\author{
Effectiveness of Partial Caries Removal in Primary Molars \\ with Deep Carious Lesions. Randomized Clinical Trial
}

\author{
Jaime A. Díaz'; Alejandra Jans ${ }^{1} \&$ Carlos Zaror ${ }^{1,2,3}$
}

DÍAZ, J. A. ; JANS, A. \& ZAROR, C. Efectividad de la remoción parcial de caries en molares primarios con lesiones de caries profunda. Ensayo clínico aleatorizado. Int. J. Odontostomat., 11(4):443-449, 2017.

RESUMEN: Para el tratamiento de lesiones de caries profundas la remoción parcial de caries (RPC) parece presentar ventajas por sobre la remoción completa de caries (RCC). Sin embargo, aún la evidencia es insuficiente para determinar si hay diferencias en relación a signos y síntomas del complejo dentino-pulpar entre ambos tratamientos. El objetivo de este estudio fue determinar la efectividad de la remoción parcial de caries en lesiones de caries dentinarias activas profundas de molares primarios. Se realizó un ensayo clínico controlado aleatorizado de 2 brazos paralelos en 20 participantes entre 4 y 8 años. Los participantes fueron asignado aleatoriamente recibir remoción parcial de la lesión de caries mientras que el grupo control recibió remoción completa de la lesión de caries. La variable de resultado primaria fue éxito del tratamiento medido como la la ausencia de cualquier signo clínico y radiográfico de patología pulpar. Un enfoque de intención de tratar (ITT) se utilizó para el análisis primario y la tasa de supervivencia de cada tratamiento se estimó mediante análisis de Kaplan-Meier. Treinta y ocho dientes fueron incluidos en el estudio. Diecisiete dientes recibieron RPC y $21 \mathrm{RCC}$. El seguimiento promedio fue de 12,6 DE 5,0 meses para ambos grupos. La tasa de éxito clínico fue de un 94,1\% para la RPC y de $76,2 \%$ para la RCC ( $p=0,132)$. La sobrevida estimada a los 18 meses fue de un $92,3 \%$ para RPC y de un $75,9 \%$ para la RCC ( $p>0,05)$. En conclusión, a pesar que la terapia RPC presentó menos complicaciones, no mostró ser más efectiva que la RCC en lesiones de caries dentinarias profundas de dientes primarios.

PALABRAS CLAVE: caries dental, ensayo clínico, remoción parcial, dientes primarios.

\section{INTRODUCCIÓN}

La caries dental corresponde a un proceso dinámico que se desarrolla en el biofilm dental como resultado de un desequilibrio entre el esmalte dentario y los depósitos microbianos (Fejerskov, 1997). Una vez producida la cavitación por el avance de la lesión, diferentes tipos de intervenciones clínicas en el campo de la odontología restauradora y terapia pulpar han sido propuestas y desarrolladas para detener la progresión hacia el complejo dentino pulpar, y/o evitar complicaciones infecciosas a nivel pulpar (Fejerskov; Kidd, 2004).
El enfoque terapéutico convencional de la caries ha sido el proceso restaurador quirúrgico invasivo y no conservador, en el cual consiste en la remoción con instrumentos manuales y rotatorios de la totalidad de la dentina reblandecida y desmineralizada, tanto en dientes primarios como permanentes (Weerheijm \& Groen, 1999).

Sin embargo, el escenario clínico es complejo, sobre todo cuando intervenimos con esta filosofía dientes asintomáticos con lesiones dentinarias profundas

${ }^{1}$ Programa de Especialización en Odontología Pediatrica, Departamento de Odontopediatría y Ortodoncia, Facultad de Odontología, Universidad de La Frontera, Temuco, Chile

${ }^{2}$ Centro de Investigación en Epidemiología, Economía y Salud Públcia Oral (CIEESPO), Facultad de Odontología, Universidad de La Frontera, Temuco, Chile

${ }^{3}$ Facultad de Odontología, Universidad San Sebastían, Puerto Montt

Este estudio fue financiado por la Universidad de La Frontera, Proyecto DIUFRO Nº DI12-0021 
activas en dientes primarios y dientes permanentes jóvenes, lo cual comúnmente implica mayor riesgo de exposición pulpar (Magnusson \& Sundell, 1977; Leksell et al., 1996; Ricketts et al., 2013).

En los últimos años, esta visión del tratamiento ha sido modificado como resultado de la mejor comprensión y conocimiento del proceso de caries; y actualmente se ha propuesto y aceptado una aproximación de mínima invasión en la cual se mantiene la porción más interna de dentina cariada que contiene colágeno intacto y que es posible de remineralizar. Existen varias aproximaciones terapéuticas de mínima invasión, siendo la primera de ellas la técnica en dos pasos o "stepwise", en donde en una primera sesión la lesión de caries es removida parcialmente manteniendo tejido cariado en la proximidad de la pared pulpar y sellando con un material provisorio. En una segunda visita, meses después, se realiza la reapertura de la cavidad y se elimina completamente el tejido cariado remanente sellando con una restauración definitiva. Esto permitiría el desarrollo de dentina terciaria entre las dos sesiones (Bjørndal et al., 1997, 2010). Basado en múltiples reportes en relación a la variada respuesta del proceso de caries y del complejo dentino pulpar posterior al sellado del tejido cariado, se ha discutido sobre la necesidad de re-entrar y realizar la remoción completa de la dentina cariada subyacente (Ricketts et al., 2006; Thompson et al., 2008).

Evidencia ha demostrado que tanto la remoción completa de caries (RCC) o una segunda visita de en la técnica stepwise para remover todo el tejido cariado aumenta la probabilidad de exposición pulpar y sus complicaciones (Ricketts et al., 2006; Maltz et al., 2012; Ricketts et al., 2013). Es por eso que la remoción parcial de caries (RPC), en donde solo la dentina necrótica desorganizada es removida, y el diente es restaurado en forma definitiva en la misma sesión, a mostrado buenos resultados en relación a disminución del riesgo de exposición pulpar. Además reduce el tamaño de la cavidad con la consecuente preservación de la estructura dentaria.

Sin embargo, aún la evidencia es insuficiente para determinar si hay diferencias en relación a signos y síntomas de enfermedad pulpar entre la técnica de RPC versus RCC (Maltz et al., 2002; Oliveira et al., 2006; Maltz et al., 2007).

El objetivo de este ensayo clínico controlado fue evaluar la efectividad de la remoción parcial de caries en comparación a la remoción completa en lesiones de caries dentinarias profundas de molares primarios.

\section{MATERIAL Y MÉTODO}

Se realizó un ensayo clínico controlado aleatorizado por conglomerado de 2 brazos paralelos entre Julio del 2012 y Abril del 2014 para evaluar la efectividad de la remoción parcial de caries en comparación a la remoción completa de la lesión de caries en molares primarios. El protocolo del estudio fue aprobado por el Comité Ético Científico de la Universidad de La Frontera con resolución nº 011/2014.

El estudio fue conducido en dependencias de la clínica de la especialidad de Odontopediatría, Universidad de La Frontera, Temuco, Chile. Se invitaron a participar niños entre 4-8 años que presentaran molares primarios con caries dentinarias profundas activas próxima a tejido pulpar (ICDAS $4,5,6$ ) que radiográficamente comprometan al menos el $50 \%$ del espesor de la dentina, asintomáticos o con sintomatología compatible con inflamación pulpar reversible, y cuyos padres hayan autorizado su participación en el estudio mediante la firma de un formulario de consentimiento. Fueron excluidos molares primarios con alteraciones de la estructura dentaria, con destrucción coronaria extensa que impida su rehabilitación y aquellos molares con signos de necrosis pulpar, fístula activa, y molares primarios que al examen radiográfico presenten radiolucidez periradicular y/o presencia de endorizalisis o exorizalisis. La Figura 1 muestra el aspecto clínico y radiográfico de las lesiones tratadas.

Descripción de las intervenciones. Todos los investigadores fueron actualizados y entrenados antes de comenzar el estudio. La RPC se realizó con cucharetas eliminando dentina reblandecida infectada del fondo y paredes cavitarias. La cavidad se lavó con agua destilada y se secó con motitas de algodón, sin aplicar aire a presión de la jeringa triple del equipo (Fig. 2).

La RCC del fondo cavitario y de sus paredes se realizó con fresas de carbide redondas y cuchareta de caries. Se realizó el tratamiento pulpar indirecto o directo con cemento de hidróxido de Calcio (Dycal ${ }^{\circledR}$, Dentsply, USA) cuando fue necesario. En ambos grupos, se realizó el sellado de la cavidad con vidrio ionómero de alta viscosidad (Ketac Molar $®$, 3M ESPE, USA). Después de una semana, se realizaron restauraciones con composite o coronas pediátricas $\mathrm{Ni}$ $\mathrm{Cr}$ (3M ESPE, USA) sin eliminar el sellado primario de acuerdo a la magnitud de la destrucción coronaria. 


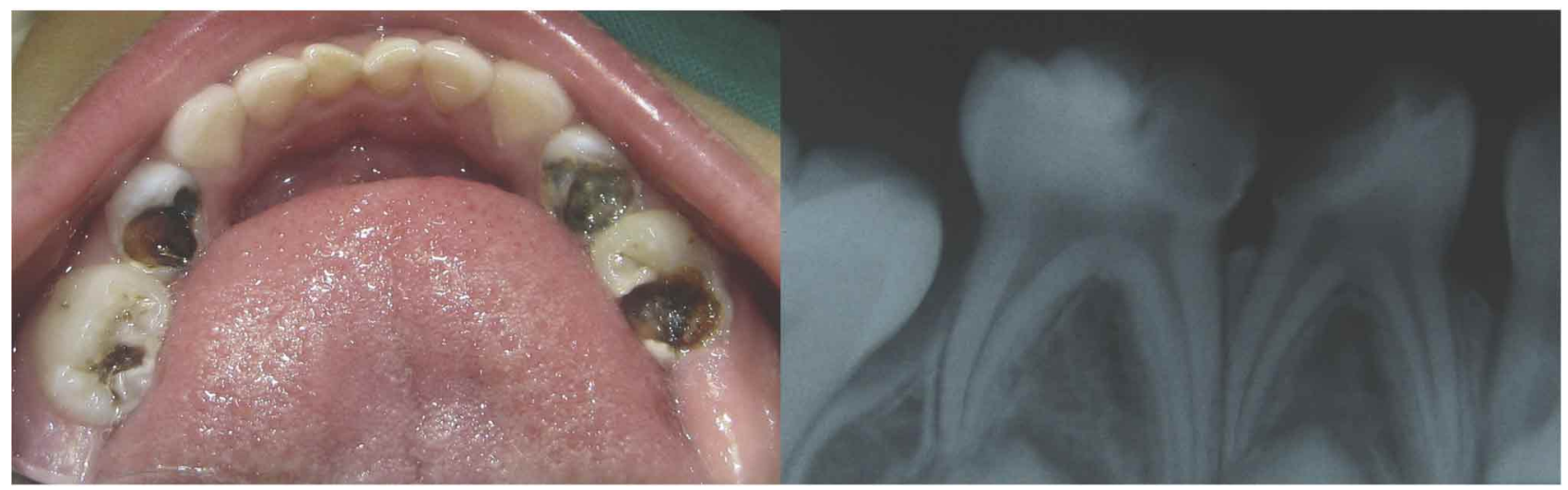

Fig. 1. Aspecto clínico y radiográfico de las lesiones de caries tratadas.

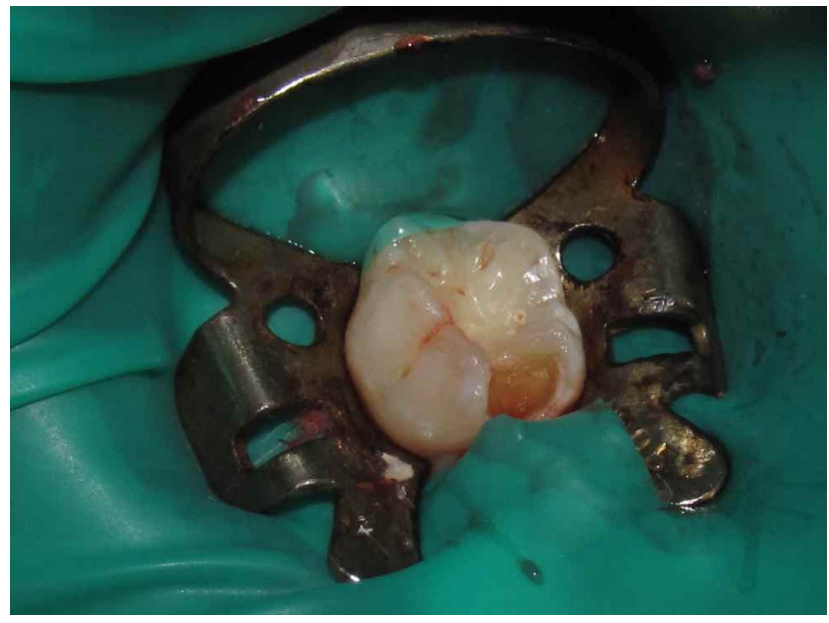

Fig. 2. Diente 7.5 sometido a tratamiento de remoción parcial de caries.

Los dientes fueron evaluados clínica y radiográficamente cada 3 meses, por dos investigadores previamente calibrados ajenos a la etapa de tratamiento y con desconocimiento del grupo de intervención asignado para cada molar.

El tamaño de la muestra se calculó para detectar diferencias de por lo menos 40 puntos porcentuales en las tasas de éxito entre los dos tratamientos a favor del protocolo con remoción parcial de caries y asumiendo una tasa de fracaso de un $50 \%$ entre los molares con caries activas profundas que recibieron tratamiento convencional (Ricketts et al., 2006). Lo anterior sumado a un nivel de significación de $5 \%$ y un poder estadístico de $80 \%$, nos llevo a reclutar un tamaño de muestra mínimo suficiente de 27 molares por grupo. Stata 13 (Stata Corp, College Station, TX, USA) se utilizó para calcular el tamaño de la muestra. El listado de la aleatorización fue generado por medio de software. El investigador involucrado en este proceso no participó en la recolección de datos o la evaluación de pacientes. Un asistente que no participó en el estudio de campo preparó el orden de los tratamientos y numeró secuencialmente los sobres sellados de acuerdo con la lista de asignación al azar. El clínico a cargo de la intervención estaba ciego a este listado hasta el inicio del tratamiento.

El grupo experimental recibió remoción parcial de la lesión de caries mientras que el grupo control recibió remoción completa de la lesión de caries. En el caso que un participante, presentara otra lesión de caries que cumpla con los criterios de selección establecidos recibió el mismo tratamiento al que fue asignado el primer molar.

La variable de resultado primaria fue éxito del tratamiento medido como la la ausencia de dolor espontáneo y/o a la percusión, de fístula, aumento de volumen y movilidad patológica y de cambios patológicos periradiculares y radiculares.

Análisis estadístico. Un enfoque de intención de tratar (ITT) se utilizó para el análisis primario. Este enfoque incluyó la asignación al azar de todos los participantes independientemente de los sucesos posteriores; por lo tanto, se incluyeron en el análisis los últimos datos registrados para medir el éxito. Se calcularon porcentajes para las variables categóricas y las medias y desviaciones estándar para las variables continuas. Los grupos se compararon utilizando la prueba t de Student para variables continuas y la prueba de chi cuadrado para variables categóricas. El nivel de significación para todas las pruebas se estableció como $p<0,05$. La tasa de supervivencia de cada tratamiento se estimó mediante análisis de Kaplan-Meier y la prueba log-rank. Los datos de análisis se realizaron utilizando Stata 13 (Stata Corp, College Station, TX, USA). 


\section{RESULTADOS}

Treinta y ocho dientes en 20 participantes entre 4 y 8 años $(5,55 \pm D E 1,19)$ fueron incluidos en el estudio. Diecisiete dientes recibieron RPC y 21 RCC. No hubo diferencia entre los grupos en término de edad, sexo, nivel socioeconómico e índice ceod. El seguimiento promedio fue de $12,6 \pm$ DE 5,0 meses para ambos grupos, siendo de $13,2(\mathrm{de}=4,4)$ meses para la RPC y de $12,1(\mathrm{de}=5,5)$ meses para RCC. La tasa de éxito clínico fue de un 94,1\% para la RPC y de 76,2 \% para la RCC $(p=0,132)$. En todos los casos las causas de los fracasos fueron abscesos dentoalveolares. Los 5 casos de fracasos en el grupo RCC fallaron durante los primeros 6 meses de seguimiento y el único fracaso del grupo experimental se presentó a los 12 meses. El riesgo relativo fue de 0,2 con $\mathrm{Cl} 195 \%$ de $0,03-1,91$. NNT fue de $6(\mathrm{Cl} 95 \%-14 \mathrm{a} \infty)$

La sobrevida estimada a los 18 meses fue de un $92,3 \%$ para RPC y de un $75,9 \%$ para la RCC $(p>0,05)$ (Fig. 3).

\section{REVIDA}

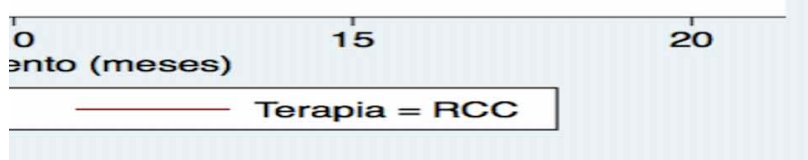

Fig. 3. Sobrevida acumulada para las terapias de RPC y RCC.

\section{DISCUSIÓN}

Este ensayo clínico controlado y aleatorizado comparó la efectividad de la remoción parcial de caries con la remoción completa de caries en lesiones profundas de molares primarios. El enfoque por ITT no encontró diferencias significativas entre los grupos en cuanto a signos y síntomas de patología pulpar, sin embargo una diferencia clínica de un $18 \%$ a favor de la RPC fue observada.
Nuestros hallazgos concuerdan con lo reportado por Franzon et al. (2014) donde las tasas de éxito clínico y radiográfico de ambos tratamiento en dientes primarios con lesiones cariosas profundas fueron altas y no difirieron significativamente. Además concluyen que la RPC es un abordaje mínimamente invasivo fiable en los dientes primarios y que la retención de la dentina cariada no interfiere con la vitalidad pulpar. Destacan que a pesar de no haber diferencias significativas entre los grupos, la RPC proporcionó otras ventajas clínicamente relevantes sobre la RCC, como son una menor incidencia de exposición a la pulpa y un menor tiempo operatorio.

Phonghanyudh et al. (2012) comparó la RPC con el Tratamiento Restaurador Atraumático y la RCC. Sus resultados tampoco mostraron diferencias entre los grupos después de 12 meses de evaluación clínica y radiográfica.

Por su parte, Franzon et al. (2015) mostró que si bien la RPC previene la exposición a la pulpa y, en consecuencia, tratamientos más invasivos, esta presentó menor longevidad para la restauración de resina compuesta en comparación con RCC, lo que sugiere que las restauraciones realizadas con RPC deben seguirse con el tiempo, especialmente en restauraciones multisuperficie.

Dado que en nuestro estudio los resultados de los tratamientos se comportan de manera similar, lo primero nos debemos preguntar: ¿Es necesario eliminar todo el tejido cariado de las lesiones profundas en dentina cercanas a la pulpa?.

Desde la época de GV Black, la remoción completa de caries ha sido realizada como el "gold standard", en lo que respecta a la preparación de cavidades y como tratamiento adecuado de los dientes afectados por la caries, y que a pesar de la falta de evidencia, se mantiene aún como una práctica común entre los cirujano-dentistas. Existen incluso estudios publicados que hacen referencia a encuestas realizadas a dentistas, en las cuales la mayoría de ellos declara que realizan la eliminación completa del tejido cariado, incluso si el procedimiento involucra riesgo de perforación pulpar (Oen et al., 2007; Schwendicke et al., 2013).

Hasta nuestros tiempos, el tratamiento de las lesiones de caries profundas en dentina sigue siendo un gran desafío para la odontología, especialmente en dentición permanente joven y en dientes primarios, toda vez que estos, por su particular anatomía e histofisiología 
pulpar representan un alto riesgo de inflamación pulpar aguda y perforación pulpar accidental durante el procedimiento (Magnusson \& Sundell; Leksell et al.).

Entonces, una de las mayores áreas de interés y discusión en relación al manejo clínico de lesiones de caries dentinarias profundas, se refiere a la real efectividad del tratamiento restaurador, especialmente al comportamiento clínico y manifestación radiográfica del complejo dentino pulpar.

Una de las mejores evidencias que fundamentan la remoción parcial de caries en dientes con lesiones profundas en dentina, son aquellos estudios relacionados con la técnica stepwise. La literatura en relación a esta terapia ha reportado en forma consistente que la dentina residual se "endurece" bajo restauraciones temporales (Bjørndal et al., 1997). Entonces, si uno de los objetivos centrales es evitar la exposición pulpar, ¿porque someter al paciente a mayor riesgo de perforación e inflamación pulpar?.

Como ha sido ampliamente reportado, la remoción completa de la dentina infectada, afectada y desmineralizada, implica mayores cambios inflamatorios agudos al tejido pulpar, mayor riesgo de una exposición pulpar, mayor probabilidad de contaminación bacteriana y la perdida de vitalidad del diente. En ese contexto es interesante considerar otros factores que pueden pronosticar un resultado negativo a nivel pulpar posterior al procedimiento convencional de eliminación completa de caries: (i) Estado de las fresas, (ii) esterilización previa de las fresas, (iii) presión ejercida por el instrumental rotatorio sobre la dentina cariada, (iv) tiempo de aplicación del fresado, (v) refrigeración adecuada si es que se utiliza turbinas de alta velocidad, (vi) grosor remanente de la dentina que conforma el techo pulpar, (vii) etapa del diente en cuanto a su ciclo vital, (viii) envejecimiento pulpar, (ix) número de superficies comprometidas y $(\mathrm{x})$ experiencia del operador.

Claramente, al coexistir múltiples factores que en mayor o menor proporción pueden influir negativamente la respuesta pulpar frente al procedimiento de remoción completa de caries, es muy improbable esperar un resultado clínico óptimo en el corto, mediano y largo plazo. En ese contexto, nuestro estudio es coherente, en el sentido de que todos los dientes con complicaciones pulpares asociadas al desarrollo de una necrosis pulpar, se manifestaron clínica y/o radiográficamente entre los 3 y 6 meses post intervención.
El número de superficies comprometidas y restauradas ha demostrado ser un fuerte indicador de fracaso, que potencialmente puede llevar irreversiblemente a una necrosis pulpar (Bjørndal et al., 2010). En nuestra experiencia, todos los casos que fracasaron con el desarrollo de necrosis pulpar y el desarrollo de abscesos dentarios fueron dientes que presentaban dos y más superficies comprometidas.

Otro factor a considerar para preferir la elección de la RPC por sobre la RCC es que actualmente existen varios resultados de ensayos clínicos han demostrado que tratamientos "conservadores" de dientes con pulpa expuesta resultan en un peor pronóstico (Barthel et al., 2000).

El desarrollo de esta "nueva odontología" también ha sido favorecida por el advenimiento de nuevos biomateriales restauradores que han permitido que el diseño cavitario sea más conservador (Featherstone \& Doméjean, 2012).

Por otro lado, para el tratamiento de caries en dientes primarios en niños, los procedimientos terapéuticos debieran ser simples, sin provocar dolor ni stress al paciente, manteniendo una adecuada respuesta pulpar, y garantizando que el diente tratado permanezca asintomático durante su ciclo vital remanente (van Amerongen \& Rahimtoola, 1999; Santamaria et al., 2015). La remoción parcial de caries podría ser una alternativa más amigable al tratamiento restaurador de la lesión de caries, sin embargo, la evidencia no es concluyente.

Últimamente con el fin de unificar los criterios de los procedimientos para la intervención de caries profundas en dentina, la International Caries Consensus Collaboration (ICCC-2016), definió los principios básicos a considerar para la remoción parcial de caries, en los cuales destaca: (i) la preservación máxima del tejido dentario, (ii) mantener la vitalidad pulpar, (iii) evitar la exposición pulpar, (iv) disminuir y evitar la ansiedad del paciente, especialmente en el niño, y (v) permitir márgenes cavitarios sanos para asegurar un buen sellado periférico de la restauración (Innes et al., 2016).

La mayor limitación de nuestro estudio fue su baja potencia, lo cual hace nuestras conclusiones inciertas. La baja potencia se debió primero por que no fuimos capaces de reclutar el mínimo de participantes requeridos de acuerdo al tamaño muestral calculado previo al inicio del estudio. Por otro lado, el seguimiento de los participantes fue incompleto e irregular, con al- 
gunos participantes que sólo presentaron 3 meses de seguimiento lo cual pudo ser insuficiente para que se presente algunos de los resultados evaluados. Creemos que esto fue influenciado por las características sociodemográficas de la muestra.

Nuestros hallazgos invitan a realizar ensayos clínicos a largo plazo que no sólo evalúen parámetros clínicos sino también que evalúen aspectos importantes en la toma de decisión clínica y desde la perspectiva del paciente como los son la calidad de vida, la incomodidad durante el procedimiento o la satisfacción con el tratamiento recibido.

En conclusión, si bien en esta investigación la RPC presenta menos complicaciones durante el seguimiento que la RCC, este estudio no presenta antecedentes para sostener que la RPC es más efectiva que la RCC en lesiones dentinarias profundas de dientes primarios.

DÍAZ, J. A. ; JANS, A. \& ZAROR, C. Effectiveness of partial caries removal in primary molars with deep carious lesions. Randomized clinical trial. Int. J. Odontostomatol., 11(4):443449, 2017.

ABSTRACT: For the treatment of deep carious lesions, partial caries removal (PCR) appears to be advantageous over complete caries removal (CCR). However, and in light of insufficient evidence it is difficult to determine if there are differences between both treatments in relation to signs and symptoms of the dentin-pulp complex. The aim of this study was to determine the effectiveness of partial caries removal in deep carious lesions of primary molars. A randomized two-arm parallel controlled trial was performed in 20 participants, 4 to 8 years of age. Participants were randomly assigned to receive partial removal of carious lesions, while the control group was submitted to complete carious lesion removal. The primary outcome variable of treatment success was measured as absence of any clinical and radiographic signs of pulpal pathology. An intention-to-treat (ITT) approach was used for the primary analysis, and the survival rate of each treatment was estimated using Kaplan-Meier analysis. Thirty-eight teeth were included in the study. Seventeen teeth received PCR and 21 CCR. The mean follow-up was $12.6 \pm$ SD 5.0 months for both groups. The clinical success rate was $94.1 \%$ for the PCR and $76.2 \%$ for the CCR $(p=0.132)$. The estimated survival at 18 months was $92.3 \%$ for PCR and $75.9 \%$ for CCR ( $p>0.05$ ). In conclusion and according with this research, PCR did not show to be more effective than the CCR in treatment of deep carious lesions of primary teeth. However, the trend in the results favoring the PCR treatment could justify a future research to confirm or refute our results.

KEY WORDS: dental caries; clinical trial; partial excavation; primary teeth.

\section{REFERENCIAS BILIOGRÁFICAS}

Barthel, C. R.; Rosenkranz, B.; Leuenberg, A. \& Roulet, J. F. Pulp capping of carious exposures: treatment outcome after 5 and 10 years: a retrospective study. J. Endod., 26(9):525-8, 2000.

Bjørndal, L.; Larsen, T. \& Thylstrup, A. A clinical and microbiological study of deep carious lesions during stepwise excavation using long treatment intervals. Caries Res., 31(6):411-7, 1997.

Bjørndal, L.; Reit, C.; Bruun, G.; Markvart, M.; Kjaeldgaard, M.; Näsman, P.; Thordrup, M.; Dige, I.; Nyvad, B.; Fransson, H.; Lager, A.; Ericson, D.; Petersson, K.; Olsson, J.; Santimano, E. M.; Wennström, A.; Winkel, P. \& Gluud, C. Treatment of deep caries lesions in adults: randomized clinical trials comparing stepwise vs. direct complete excavation, and direct pulp capping vs. partial pulpotomy. Eur. J. Oral Sci., 118(3):290-7, 2010.

Featherstone, J. D. \& Doméjean, S. Minimal intervention dentistry: part 1. From 'compulsive' restorative dentistry to rational therapeutic strategies. Br. Dent. J., 213(9):4415, 2012.

Fejerskov, O. Concepts of dental caries and their consequences for understanding the disease. Community Dent. Oral Epidemiol., 25(1):5-12, 1997.

Franzon, R.; Guimarães, L. F.; Magalhães, C. E.; Haas, A. N. \& Araujo, F. B. Outcomes of one-step incomplete and complete excavation in primary teeth: a 24-month randomized controlled trial. Caries Res., 48(5):376-83, 2014.

Franzon, R.; Opdam, N. J.; Guimarães, L. F.; Demarco, F. F.; Casagrande, L.; Haas, A. N. \& Araujo, F. B. Randomized controlled clinical trial of the 24-months survival of composite resin restorations after one-step incomplete and complete excavation on primary teeth. $J$. Dent., 43(10):1235-41, 2015.

Innes, N. P.; Frencken, J. E.; Bjørndal, L.; Maltz, M.; Manton, D. J.; Ricketts, D.; Van Landuyt, K.; Banerjee, A.; Campus, G.; Doméjean, S.; Fontana, M.; Leal, S.; Lo, E.; Machiulskiene, V.; Schulte, A.; Splieth, C.; Zandona, A. \& Schwendicke, F. Managing carious lesions: Consensus recommendations on terminology. Adv. Dent. Res., 28(2):49-57, 2016.

Kidd, E. A. How 'clean' must a cavity be before restoration? Caries Res., 38(3):305-13, 2004.

Leksell, E.; Ridell, K.; Cvek, M. \& Mejàre, I. Pulp exposure after stepwise versus direct complete excavation of deep carious lesions in young posterior permanent teeth. Endod. Dent. Traumatol., 12(4):192-6, 1996.

Magnusson, B. O. \& Sundell, S. O. Stepwise excavation of deep carious lesions in primary molars. J. Int. Assoc. Dent. Child., 8(2):36-40, 1977.

Maltz, M.; de Oliveira, E. F.; Fontanella, V. \& Bianchi, R. A clinical, microbiologic, and radiographic study of deep caries lesions after incomplete caries removal. Quintessence Int., 33(2):151-9, 2002.

Maltz, M.; Garcia, R.; Jardim, J. J.; de Paula, L. M.; Yamaguti, 
P. M.; Moura, M. S.; Garcia, F.; Nascimento, C.; Oliveira, A. \& Mestrinho, H. D. Randomized trial of partial vs. stepwise caries removal: 3-year follow-up. J. Dent. Res., 91(11):1026-31, 2012.

Maltz, M.; Oliveira, E. F.; Fontanella, V. \& Carminatti, G. Deep caries lesions after incomplete dentine caries removal: 40-month follow-up study. Caries Res., 41(6):493-6, 2007.

Oen, K. T.; Thompson, V. P.; Vena, D.; Caufield, P. W., Curro, F.; Dasanayake, A.; Ship, J. A. \& Lindblad, A. Attitudes and expectations of treating deep caries: a PEARL Network survey. Gen. Dent., 55(3):197-203, 2007.

Oliveira, E. F.; Carminatti, G.; Fontanella, V. \& Maltz, M. The monitoring of deep caries lesions after incomplete dentine caries removal: results after 14-18 months. Clin. Oral Investig., 10(2):134-9, 2006.

Phonghanyudh, A.; Phantumvanit, P.; Songpaisan, Y. \& Petersen, P. E. Clinical evaluation of three caries removal approaches in primary teeth: a randomised controlled trial. Community Dent. Health, 29(2):173-8, 2012.

Ricketts, D. N.; Kidd, E. A.; Innes, N. \& Clarkson, J. Complete or ultraconservative removal of decayed tissue in unfilled teeth. Cochrane Database Syst. Rev., (3):CD003808, 2006.

Ricketts, D.; Lamont, T.; Innes, N. P.; Kidd, E. \& Clarkson, J. E. Operative caries management in adults and children. Cochrane Database Syst. Rev., (3):CD003808, 2013.

Santamaria, R. M.; Innes, N. P.; Machiulskiene, V.; Evans, D. J.; Alkilzy, M. \& Splieth, C. H. Acceptability of different caries management methods for primary molars in a RCT. Int. J. Paediatr. Dent., 25(1):9-17, 2015.

Schwendicke, F.; Meyer-Lueckel, H.; Dörfer, C. \& Paris, S. Attitudes and behaviour regarding deep dentin caries removal: a survey among German dentists. Caries Res., 47(6):566-73, 2013.

Thompson, V.; Craig, R. G.; Curro, F. A.; Green, W. S. \& Ship, J. A. Treatment of deep carious lesions by complete excavation or partial removal: a critical review. J. Am. Dent. Assoc., 139(6):705-12, 2008.

van Amerongen, W. E. \& Rahimtoola, S. Is ART really atraumatic? Community Dent. Oral Epidemiol., 27(6):4315, 1999.

Weerheijm, K. L. \& Groen, H. J. The residual caries dilemma. Community Dent. Oral Epidemiol., 27(6):436-41, 1999.

\author{
Dirección para correspondencia: \\ Dr. Jaime A. Díaz \\ Departamento de Odontopediatría y Ortodoncia \\ Facultad de Odontología \\ Universidad de La Frontera \\ Manuel Montt $112,4^{\circ}$ piso \\ Temuco \\ CHILE \\ E-mail : jaime.diaz@ufrontera.cl
}

Recibido : 17-08-2017

Aceptado: 28-08-2017 